\title{
Anterolateral rupture of popliteal cysts in rheumatoid arthritis
}

\author{
B Kirkham, M Churchill, B Dasgupta, L Wedderburn, J Spencer, D G Macfarlane
}

\begin{abstract}
Popliteal cysts occur commonly in both normal and arthritic knees. Most cysts are formed by distension of the medially situated semimembranosus bursa. Popliteus bursa distension occurs uncommonly as a lateral popliteal cyst. Two cases of rupture of lateral cysts which produced symptoms related to the anterolateral lower leg are reported. The difficulty of diagnosing the condition because of this unusual site of inflammation and subsequent management problems are discussed.
\end{abstract}

Popliteal cyst rupture into the calf muscles causing the 'pseudothrombophlebitis' syndrome $^{12}$ is well described in patients with osteoarthritis and inflammatory arthritis, ${ }^{3-6}$ and has been reported in patients without preexisting knee disease. ${ }^{7}$ We report two cases that illustrate rupture of the uncommon lateral popliteal cyst into the anterolateral lower leg.

\section{Case reports}

CASE 1

A 68 year old man with a four year history of seropositive rheumatoid arthritis presented with a three day history of a painful swollen left shin. His arthritis was active, and both knees had

Accepted for publication 1 March 1990

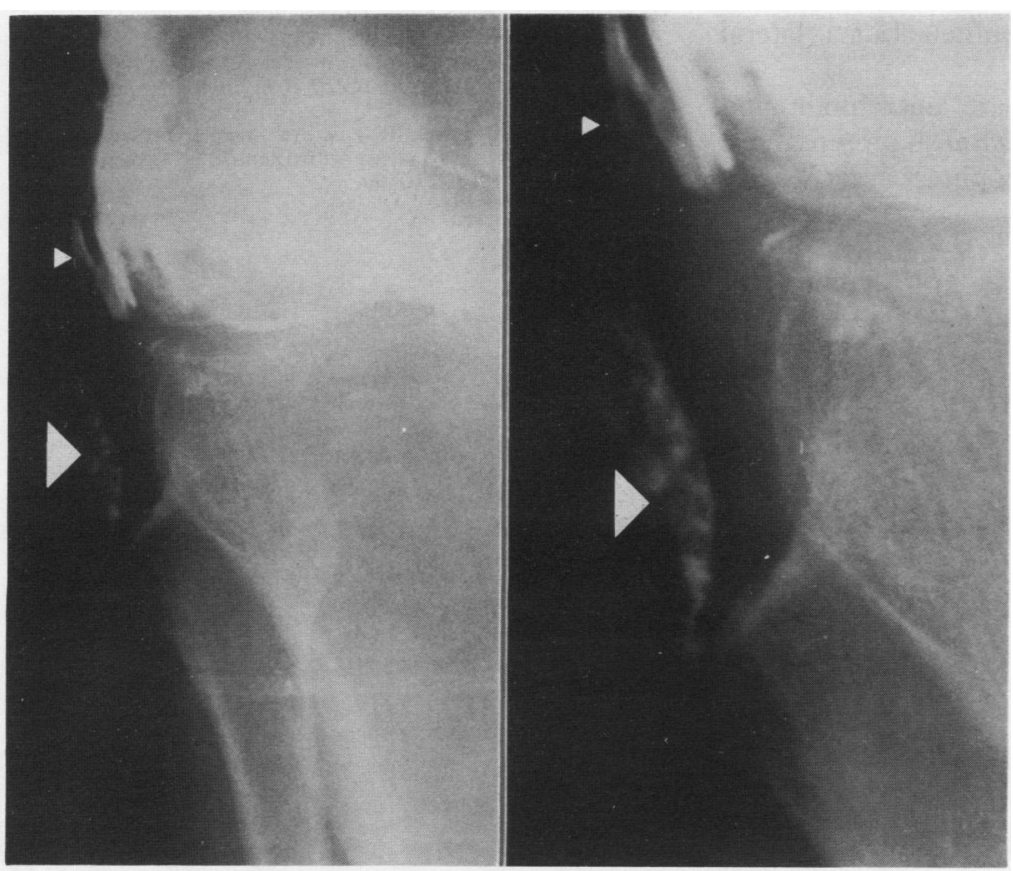

Arthrogram of left knee demonstrating the communication (small arrow) between the popliteus bursa and the wound site (large arrow). recently been aspirated and injected with methylprednisolone acetate. Treatment was with azathioprine $150 \mathrm{mg}$ daily, prednisolone 5 $\mathrm{mg}$ daily, naproxen $500 \mathrm{mg}$ twice a day. He had no fever but had signs of active arthritis affecting his hand and wrist joints. The left knee was slightly warm with a synovial effusion. The left anterolateral lower leg had an extensive area of erythema and oedema, which was very tender and extended from just below the lateral knee to the ankle. The calf was soft and nontender. Localised fluctuation was felt in the upper part of the swelling, and aspiration produced $3 \mathrm{ml}$ of yellow fluid, which contained many neutrophils and was subsequently sterile on culture. Plain radiograph of the tibia and fibula was normal. A provisional diagnosis of bacterial cellulitis was made, and intravenous ampicillin and flucloxacillin were given. Over the next three days fever developed and the fluctuant area became more prominent. An ultrasound scan of this area showed a long echogenic collection of fluid $1 \mathrm{~cm}$ below the skin. Repeat needle aspiration was unproductive, and open surgical drainage was performed. At operation $30 \mathrm{ml}$ of fluid containing numerous 'melon bodies' was released. The cavity lay in a subcutaneous plane, measured $7 \times 4 \times 3 \mathrm{~cm}$, and extended superiorly and medially above the superior tibiofibular joint and behind the knee joint. An arthrogram confirmed the communication between the synovial compartment and the cavity. The communication was $1 \mathrm{~mm}$ in diameter and $2 \mathrm{~cm}$ in length and extended from the lateral part of the popliteus bursa to the superior part of the cavity. A small Baker's cyst (semimembranosus bursa) was also present (figure). After the operation large amounts of fluid drained from the operation site until $40 \mathrm{mg}$ methylprednisolone acetate was injected into the knee. The operation site then healed slowly by granulation without complication.

CASE 2

A 66 year old woman with a six year history of seropositive rheumatoid arthritis presented with a two week history of a painful swelling below her anterolateral left knee. Her arthritis had started in her left knee, later affecting the other knee and both hands. At the time of presentation her rheumatoid arthritis was inactive except for persistent synovitis in the left knee. She took indomethacin $25 \mathrm{mg}$ twice daily. On examination a $5 \times 4 \mathrm{~cm}$ swelling lateral to and below the left tibial tuberosity was noted. This area was red, warm, and oedematous. The left knee was warm with a moderate synovial effusion and 
restricted movements. The left calf was also swollen. Turbid yellow/blood stained fluid (1.5 $\mathrm{ml}$ ) was aspirated from the area of swelling and was sterile on culture. A diagnosis of anterior leak of synovial fluid was made when culture results were known to be negative. The left knee effusion was aspirated and $\mathbf{4 0}$ mg methylprednisolone acetate injected. Rapid improvement in the swelling and inflammation ensued, and at follow up three weeks later complete resolution had been achieved.

\section{Discussion}

Popliteal cysts were first described in 1840 by Adams. ${ }^{8}$ In 1877 Baker described the cyst as a distended bursa related to the semimembranosus tendon, ${ }^{9}$ and it has been suggested that the use of the name Baker's cyst be restricted to this type of popliteal cyst ${ }^{10}$ rather than applied generally to dilatations of any of the several bursae located in the popliteal fossa. These findings have been confirmed by necropsy and radiological studies, which have shown communications between the knee joint and bursae in up to $40 \%$ of normal knees at necropsy. ${ }^{11}$ The great majority of popliteal cysts are formed by fluid distension of the semimembranosus bursa, which communicates with the knee joint in some subjects. These cysts are more common in patients with any knee disease associated with increased production of synovial fluid. ${ }^{12}$ Rupture of these cysts commonly presents with popliteal and posterior calf pain and swelling, causing the pseudothrombophlebitis syndrome. ${ }^{1}{ }^{2}$ Popliteal cysts in the lateral part of the popliteal fossa are thought to be due to popliteus bursa distension ${ }^{13}$ and are much less common than semimembranosus bursa distension. It has been reported that this bursa may communicate with the superior tibiofibular joints.

The first patient had both types of popliteal cyst, but his symptoms were clearly caused by rupture of his distended popliteus bursa (lateral popliteal cyst).

Lateral popliteal cysts are uncommon and there are few reports of this clinical presentation. In an extensive review of popliteal cysts Wigley reported a single case of leakage from a lateral popliteal cyst with an arthrogram similar to that shown in the figure. ${ }^{11}$ Recently, Samanta and colleagues reported a popliteal cyst leak anterolateral to the upper tibia. ${ }^{13}$ This was a large cystic swelling from which $50 \mathrm{ml}$ of fluid was aspirated, and was clinically very different from the presentation of patient 1. Arthrography performed in their case was reported as showing contrast medium tracking by a 'circuitous route from the joint space to the swelling', and did not identify which type of popliteal cyst gave rise to the leak.

These patients illustrate the importance of making the correct diagnosis in this unusual site of a popliteal cyst rupture. Patient 2 improved rapidly after intra-articular corticosteroid treatment. In patient 1 the large amount of cellulitis and the unusual site made the diagnosis difficult and delayed appropriate treatment.

Rupture of lateral popliteal cysts produces an unusual clinical picture and failure to recognise this may delay diagnosis and appropriate treatment.

1 Prescott S M, Pearl J E, Tikoff G. "Pseudo-pseudothrombophlebitis": ruptured popliteal cyst with deep venous thrombosis. N Engl f Med 1978; 229: 1192-3.

2 Kilcoyne R F, Imray T J, Stewart E T. Ruptured Baker's cyst simulating acute thrombophlebitis. $\mathscr{J} A M A$ 1978; 240: 1517-8.

3 Bacon P A, Gerber N J. Popliteal cysts and synovial rupture in osteoarthritis. Rheumatology and Rehabilitation 1974; 13 : 98-100.

4 Hooper J C, Brookler M. Popliteal cysts and their rupture in the rheumatoid arthritic simulating thrombophlebitis. Med f A ust 1971; 1: 1371-3.

5 Richards A J. Ruptured popliteal cyst and pyogenic arthritis. BrMed F 1981; 282: 1120-1.

6 Smith D L, Bennett R M. Popliteal cyst rutpure in SLEsuperior rupture and an infected calf cyst. $\mathcal{J}$ Rheumatol superior rupture

7 Macfarlane D G, Bacon P A. Popliteal cyst rupture in normal knee joints. Br Med f 1980; 281: 1203-4.

8 Adams R. Arthritis, chronic rheumatic, of the knee joint. Dublin fournal of Medical Science 1840; 17: 520-3.

9 Baker W M. On the formation of synovial cysts in the leg in connection with disease of the knee joint. St Bartholomew's Hospital Reports 1877; 13: 245-61.

10 Taylor A R, Rana N A. A valve. An explanation of the formation of popliteal cysts. Ann Rheum Dis 1973; 32: 419-21.

11 Wigley R D. Popliteal cysts: variations on a theme of Baker. Semin Arthritis Rheum 1982; 12: 1-10.

12 Wolfe R D, Colloff B. Popliteal cysts. An arthrographic study and review of the literature. F Bone foint Surg [Am] 1972; 54: $1057-63$.

13 Samanta A, Boyd O, Roy S. An unusual presentation of Baker's cyst in a patient with rheumatoid arthritis. $B r \mathcal{F}$ Rheumatol 1988; 27: 500 . 